\title{
High serum levels of pro-brain natriuretic peptide (pro BNP) identify cardioembolic origin in undetermined stroke
}

\author{
Manuel Rodríguez-Yáñez ${ }^{\mathrm{a}}$, Tomás Sobrino ${ }^{\mathrm{a}}$, Miguel Blanco ${ }^{\mathrm{a}}$, Natalia Pérez de la Ossa ${ }^{\mathrm{b}}$, David Brea $^{\mathrm{a}}$, \\ Raquel Rodríguez-González ${ }^{\mathrm{a}}$, Rogelio Leira ${ }^{\mathrm{a}}$, Antonio Dávalos ${ }^{\mathrm{b}}$ and José Castillo ${ }^{\mathrm{a}, *}$ \\ ${ }^{a}$ Clinical Neuroscience Research Laboratory, Department of Neurology, Hospital Clínico Universitario, University \\ of Santiago de Compostela, Santiago de Compostela, Spain \\ ${ }^{\mathrm{b}}$ Department of Neurosciences, Hospital Germans Trias i Pujol, Universitat Autònoma de Barcelona, Spain
}

\begin{abstract}
Background: Stroke subtype diagnosis leads to specific therapies to reduce recurrences. Because nearly one third of patients remain with unknown etiology after a complete screening workup, we aim to investigate whether molecular markers of myocardial damage were associated with cardioembolic stroke and if they were useful to reclassify strokes of undetermined etiology.

Methods: We included 262 patients with first ischemic stroke within the first 12 hours. Stroke subtype was evaluated by TOAST criteria. Stroke of undetermined origin were reclassified into likely atherothrombotic or likely cardioembolic according to a predefined non-validated algorithm. Blood samples were obtained on admission to determine serum levels of molecular markers (pro-BNP, pro-ANP and CK-MB) of myocardial damage.

Results: Patients with cardioembolic infarct showed higher levels of pro-BNP, pro-ANP and CK-MB. Pro-BNP $>360$ pg/mL was independently associated with cardioembolic stroke (OR: 28.51, CI95\%: 5.90-136.75, $p<0.0001$ ). Stroke etiology was undetermined in 82 patients (31\%); 34 were reclassified as likely cardioembolic, 22 as likely atherothrombotic, and 26 remained as undetermined. Pro-BNP $>360 \mathrm{pg} / \mathrm{mL}$ was the only factor independently associated with likely cardioembolic stroke.

Conclusions: Pro-BNP levels higher than $360 \mathrm{pg} / \mathrm{mL}$ are associated with cardioembolic stroke and may be useful to reclassify undetermined strokes as of cardioembolic origin.
\end{abstract}

Keywords: Brain natriuretic peptide, cardioembolic stroke, ischemic stroke, ischemic stroke classification

\section{Introduction}

Stroke subtype diagnosis leads to specific therapeutic actions to reduce recurrences. TOAST criteria [1] are the most used criteria for stroke subtype classification, however, after a complete screening tool, nearly one third of patients remain without a specific etiology.

Several molecular markers, such as atrial natriuretic peptide (ANP), brain natriuretic peptide (BNP) and cre-

* Corresponding author: Prof. José Castillo, Department of Neurology, Hospital Clínico Universitario, Travesa da Choupana s/n, 15706 Santiago de Compostela, Spain. E-mail: jose.castillo@usc.es. atin kinase-MB (CK-MB), are increased in heart damage diseases, and are related with prognosis. Transient increased of CK-MB plasma levels are found in acute myocardial infarction [23], and high plasma levels of ANP and BNP have been associated with ventricular dysfunction and poor outcome in patients with acute myocardial infarction and congestive heart failure [19, 33].

These molecular markers have been also investigated in stroke patients. Some studies found that CK-MB levels increase after brain damage in absence of coronary heart disease, such as ischemic stroke, subarachnoid hemorrhage or cranial trauma $[7,10,13,21]$. High ANP levels have been found in stroke patients with 
atrial fibrillation compared to those with small vessel disease [25], and high BNP levels were found in stroke patients compared to healthy controls with similar risk factors, regardless infarct volume, stroke location and severity [9]. Recent studies found higher levels of BNP in patients with cardioembolic stroke [16,20], however, none have studied if these markers are useful to identity patients with possible cardioembolic stroke in undetermined etiology.

The objective of this study was to determine whether molecular markers of myocardial damage were associated with cardioembolic stroke and if they were useful to reclassify strokes of undetermined etiology.

\section{Patients and methods}

From January to June 2006, 372 consecutive patients with a first episode of ischemic stroke were prospectively evaluated within the first 12 hours from stroke onset. Patients in coma or with severe stroke (NIHSS $>20)(n=30)$, previous disability (defined as mRS $\geqslant 2)(n=12)$, severe systemic diseases (chronic inflammatory, infectious, or hematologic diseases, cancer and renal or liver failure) ( $n=26)$, dementia or psychiatric diseases $(n=8)$, unstable cardiovascular disease ( $n=28$ ) including acute myocardial infarct, determined by clinical, electrocardiographic and biological changes (increased levels of troponin, mioglobin and CK-MB), or life expectancy of less than 3 months $(n=6)$ were excluded. A total of 262 patients were finally included in the study. The protocol was approved by local ethics committee and informed consent was given by patients or their relatives.

Demographic data (age, sex), previous history of high blood pressure, diabetes and heart disease, alcohol consumption and smoking habit were recorded. Stroke severity on admission was evaluated by the National Institute of Health Stroke Scale (NIHSS) score. Body temperature, blood pressure, serum glucose and fibrinogen levels, chest radiography and electrocardiogram were obtained on admission. Infarct volume in diffusion-weighted magnetic resonance imaging (DWI-MRI) was obtained using a manual tracing method within the first 12 hours from stroke onset. Blood samples for further determination of molecular markers (pro-ANP, pro-BNP and CK-MB) were drawn within 24 hours after hospitalization.

History of arterial hypertension (defined as evidence of at least two blood pressure measurements $>140 / 90 \mathrm{mmHg}$ recorded on different days before stroke onset, a physician's diagnosis or use of antihypertensive treatment), diabetes (fasting serum glucose levels $\geqslant 7.0 \mathrm{mmol} / \mathrm{L}$, a physician diagnosis or use of diabetic medication), hyperlipidemia (serum cholesterol concentration $>12.2 \mathrm{mmol} / \mathrm{L}$ or use of medication), current smoking status and alcohol overuse (> $60 \mathrm{~g}$ /day) was determined at admission.

Stroke subtype was classified in atherothrombotic, cardioembolic, lacunar and undetermined according to TOAST criteria [1], once carotid and transcranial ultrasound study and echocardiogram were performed. Trained cardiologist blinded to clinical and biological data made echocardiography in patients in which no cardioembolic source was detected in electrocardiogram at admission $(n=132)$. For a secondary analysis, infarcts of undetermined etiology (both because no etiology was found after complete study or 2 causes were present) were reclassified according to the atherothrombotic and cardioembolic risk. We considered that the atherothrombotic risk was low when the patient was female, supraaortic arteries stenosis $\leqslant 50 \%$, and no vascular risk factors were present; medium atherothrombotic risk in patients with $\leqslant 2$ vascular risk factors and supraaotic arteries stenosis $\leqslant 50 \%$; and high atherothrombotic risk when supraaortic arteries stenosis $>50 \%$, subocclusive stenosis or intracranial stenosis and $>2$ vascular risk factors. Cardioembolic risk was evaluated using $\mathrm{CHADS}_{2}$ criteria [8], in which heart failure, high blood pressure, age $\geqslant 75$ years and diabetes score 1 point, and previous stroke or TIA scores 2 point. Patients with score 0 were classified as low cardioembolic risk, score 1-2 as medium risk and score 3-6 as high risk. Patients with high atherothrombotic and low cardioembolic risk were reclassified as likely atherothrombotic, and patients with high cardioembolic and low atherothrombotic risk as likely cardioembolic. Patients with medium atherothrombotic and/or cardioembolic, high cardioembolic and atherothrombotic or low cardioembolic and atherothrombotic risk remain as undetermined.

\subsection{Laboratory tests}

Blood samples on admission were collected in glass chemistry test tubes, centrifuged at $3000 \mathrm{~g}$ for 10 minutes, and immediately frozen and stored at $-80^{\circ} \mathrm{C}$. Serum pro-BNP and CK-MB levels were measured by electrochemiluminescence immunoassay "ECLIA", ELECSYS 2010 System, Roche Diagnostics GmbH, Mannheim, Germany. Finally, serum levels of pro-ANP were measured with a commercially avail- 
Table 1

Basal characteristics of patients according to stroke subtype, univariated analysis

\begin{tabular}{|c|c|c|c|c|c|}
\hline & $\begin{array}{l}\text { Atherothrombotic } \\
\quad n=44 \\
\end{array}$ & $\begin{array}{c}\text { Cardioembolic } \\
n=100 \\
\end{array}$ & $\begin{array}{l}\text { Lacunar } \\
n=36\end{array}$ & $\begin{array}{c}\text { Undetermined } \\
n=82 \\
\end{array}$ & $\mathrm{p}$ \\
\hline Age, years & $67.8 \pm 9.5$ & $72.3 \pm 11.1$ & $65.1 \pm 12.6$ & $68.1 \pm 11.7$ & 0.015 \\
\hline Female, $\%$ & 31.8 & 50.0 & 38.9 & 36.6 & 0.133 \\
\hline History of hypertension, $\%$ & 63.6 & 62.0 & 44.0 & 51.2 & 0.158 \\
\hline History of diabetes, $\%$ & 36.4 & 14.0 & 22.2 & 17.1 & 0.017 \\
\hline Hypercholesterolemia, \% & 36.4 & 18.0 & 11.1 & 26.8 & 0.024 \\
\hline Smoker, $\%$ & 31.8 & 18.0 & 22.0 & 19.5 & 0.294 \\
\hline $\begin{array}{l}\text { Alcohol consumption } \\
>40 \text { g/day, } \%\end{array}$ & 9.1 & 2.0 & 0 & 7.3 & 0.004 \\
\hline Coronary heart disease, $\%$ & 9.1 & 22.0 & 5.6 & 2.4 & $<0.0001$ \\
\hline Atrial fibrillation, $\%$ & 0 & 50.0 & 11.1 & 4.9 & $<0.0001$ \\
\hline $\mathrm{SBP}, \mathrm{mm} \mathrm{Hg}$ & $144.4 \pm 26.1$ & $151.2 \pm 22.6$ & $157.1 \pm 24.3$ & $150.9 \pm 20.0$ & 0.705 \\
\hline $\mathrm{DBP}, \mathrm{mm} \mathrm{Hg}$ & $78.6 \pm 11.7$ & $79.9 \pm 11.5$ & $85.1 \pm 16.4$ & $80.1 \pm 16.6$ & 0.705 \\
\hline Body temperature, ${ }^{\circ} \mathrm{C}$ & $36.2 \pm 0.5$ & $36.4 \pm 0.3$ & $36.3 \pm 0.3$ & $36.3 \pm 0.3$ & 0.120 \\
\hline Glucose, $\mathrm{mg} / \mathrm{dL}$ & $118.2 \pm 32.5$ & $126.1 \pm 28.7$ & $115.4 \pm 23.9$ & $131.3 \pm 49.8$ & 0.499 \\
\hline Fibrinogen, $\mathrm{mg} / \mathrm{dL}$ & $419.7 \pm 142.3$ & $443.9 \pm 145.4$ & $335.6 \pm 62.9$ & $371.2 \pm 95.2$ & 0.001 \\
\hline Basal NIHSS & $12[7,17]$ & $12[8,17]$ & $6[3,8]$ & $10[6,16]$ & $<0.0001$ \\
\hline DWI volume at admission, $\mathrm{cc}$ & $19.2[5.0,54.0]$ & $25.4[15.0,48.0]$ & $1[0,1.0]$ & $24.5[6.0,68.0]$ & $<0.0001$ \\
\hline Carotid stenosis $>50 \%, \%$ & 77.3 & 0 & 5.6 & 4.9 & $<0.0001$ \\
\hline Carotid stenosis $\leq 50 \%, \%$ & 22.7 & 15.0 & 11.1 & 22.0 & 0.346 \\
\hline Intracranial stenosis, $\%$ & 20.5 & 4.0 & 5.6 & 9.8 & 0.012 \\
\hline Pro-BNP levels (pg/mL) & $64.4[15.6,250.7]$ & $1131.5[483.9,1959.0]$ & $151.7[78.4,355.8]$ & $225.7[110.5,863.7]$ & $<0.0001$ \\
\hline Pro-ANP levels (fmol/mL) & $1020.2[604.2,2609.6]$ & $3359.4[1950.5,7250.6]$ & $1223.3[451.0,3058.1]$ & $1377.8[599.7,4868.1]$ & $<0.0001$ \\
\hline CK-MB levels (ng/mL) & $1.90[1.27,2.46]$ & $3.02[2.23,4.08]$ & $2.23[1.99,2.74]$ & $2.65[2.03,4.86]$ & $<0.0001$ \\
\hline
\end{tabular}

SBP: systolic blood pressure, DBP: diastolic blood pressure, DWI: diffusion-weighted image.

able quantitative enzyme-linked immunosorbent assay (ELISA) kits obtained from Biomedica GmbH, Vienna, Austria. Determinations were performed in an independent laboratory blinded to clinical and neuroimaging data. The intra-assay and interassay coefficients of variation were $<5 \%$ for NT-proBNP, pro-ANP and CK-MB determinations.

\subsection{Statistical analyses}

Results are expressed as percentages for categorical variables and compared using the chi-square test, and as mean (standard deviation), or median [quartiles] for the continuous variables. Comparison of baseline characteristics of the different groups was calculated by ANOVA. ROC curves were configured to establish cut-off points of molecular marker levels that optimally predicted the cardioembolic origin of stroke. Odds ratios were adjusted by significant variables in the bivariate analysis.

Values of $\mathrm{p}$ below 0.05 were considered to be statistically significant in all tests. The statistical analysis was conducted using SPSS 16.0 for Windows XP.

\section{Results}

We included 262 patients $(69.8 \pm 11.8$ years, $58.7 \%$ male). Stroke subtype was classified as atherothrom- botic in 44 patients $(16.8 \%)$, cardioembolic in 100 $(38.2 \%)$, lacunar in $36(13.7 \%)$, and undetermined in $82(31.3 \%)$.

Basal characteristics of patients are shown in Table 1. No differences in infarct volume were found between atherothrombotic and cardioembolic infarcts. Higher levels of pro-BNP, pro-ANP and CK-MB were found in patients with cardioembolic infarcts (Fig. 1).

Using ROC curves analyzing cardioembolic versus non-cardioembolic infarcts (atherothrombotic, lacunar and undetermined), we found that the best cutoff point of pro-BNP to predict cardioembolic stroke was $360 \mathrm{pg} / \mathrm{mL}$ (area under curve 0.921, sensitivity $87 \%$, specificity $83 \%, p<0.0001$ ), of pro-ANP $2266.6 \mathrm{fmol} / \mathrm{mL}$ (area under curve 0.735 , sensitivity $62 \%$, specificity $70 \%, p<0.0001$ ) and of CK-MB $2.6 \mathrm{ng} / \mathrm{mL}$ (area under curve 0.731 , sensitivity $62 \%$, specificity $80 \%, p<0.0001)$. In a logistic regression model, pro-BNP $>360 \mathrm{pg} / \mathrm{mL}$ (OR: 28.51, CI 95\%: 5.90-136.75, $p<0.0001)$ was independently associated with higher risk of cardioembolic stroke, after adjustment for significant variables in univariated analysis (Table 2).

In a further analysis of undetermined infarcts, using the above criteria, 34 patients $(41 \%)$ were reclassified as likely cardioembolic, 22 patients $(27 \%)$ as likely atherothrombotic, and 26 patients $(32 \%)$ remained 

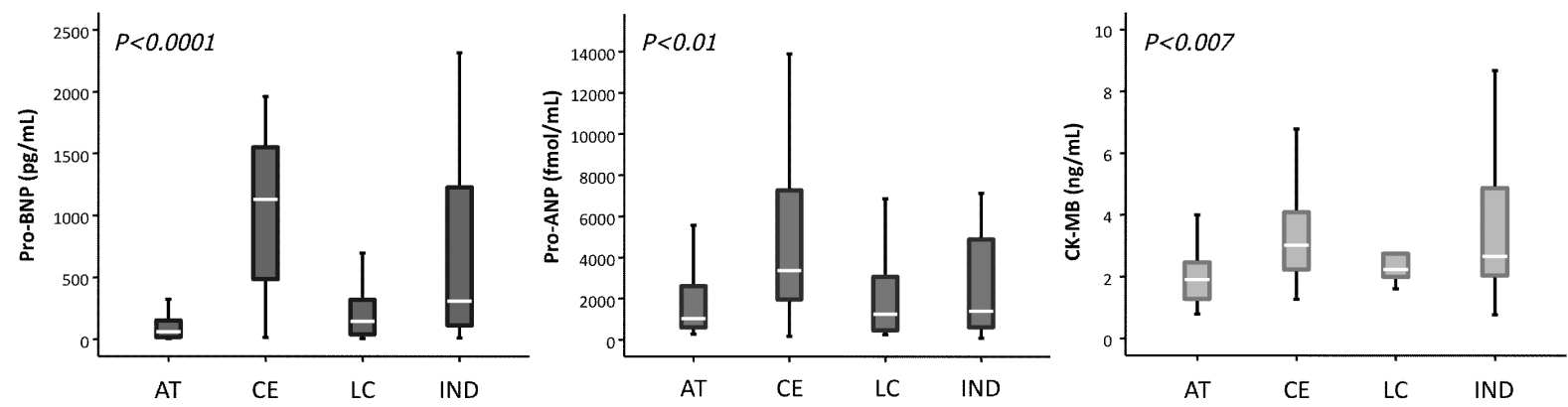

Fig. 1. Molecular markers values across the different etiological groups. AT: atherothrombotic; CE: cardioembolic; LC: lacunar; UND: undetermined.

Table 2

Logistic regression model, dependent variable cardioembolic stroke

\begin{tabular}{lrcc}
\hline & OR & CI 95\% & $\mathrm{p}$ \\
\hline Age & 0.90 & $0.81-1.14$ & 0.618 \\
Sex, female & 0.72 & $0.26-3.17$ & 0.694 \\
Diabetes & 1.10 & $0.21-7.98$ & 0.886 \\
Coronary heart disease & 2.74 & $0.51-15.46$ & 0.129 \\
Atrial fibrillation & 20.63 & $4.24-100.91$ & $<0.0001$ \\
Temperature & 1.13 & $0.86-26.40$ & 0.308 \\
Fibrinogen & 1.00 & $0.94-1.13$ & 0.166 \\
Basal NIHSS & 1.00 & $0.92-1.20$ & 0.388 \\
DWI volume & 0.93 & $0.90-1.12$ & 0.077 \\
Stenosis $>50 \%$ & 0.30 & $0.2-318.74$ & 0.904 \\
Intracranial stenosis & 0.26 & $0.10-2.74$ & 0.144 \\
Pro BNP $\geqslant 360$ & 28.51 & $5.90-136.75$ & $<0.0001$ \\
Pro ANP $\geqslant 2266.6$ & 1.26 & $0.92-1.78$ & 0.127 \\
CK-MB $\geqslant 2.6$ & 3.3 & $1.1-10.1$ & 0.032 \\
\hline
\end{tabular}

as undetermined. Basal characteristics and biomarker serum levels of these subgroups of patients are shown in Table 3. In a logistic regression model in undetermined patients, analyzing likely cardioembolic versus non-likely cardioembolic (likely atherthrombotic and undetermined), only pro-BNP $>360 \mathrm{pg} / \mathrm{mL}$ (OR: 35.8 , CI 95\%: 5.68-225.16, $p<0.0001$ ) was independently associated with likely cardioembolic stroke.

\section{Discussion}

This study shows that patients with cardioembolic stroke have higher levels of molecular markers of cardiac damage (pro-ANP, pro-BNP and CK-MB), in comparison to the other etiological subtypes of stroke. ProBNP was the most powerful molecular marker associated with cardiembolic stroke. Serum levels of pro-BNP $>360 \mathrm{pg} / \mathrm{mL}$ increased about 30 fold the probability of cardioembolic stroke in the whole group. Furthermore, pro-BNP levels were useful to reclassify undetermined strokes. Pro-BNP serum levels $>360$ pg/mL among patients with stroke of unknown etiology were found in $94.1 \%$ of those likely cardioembolic, whereas in none of those likely atherothrombotic.

BNP is a neurohormone that is synthesized and released primarily from the cardiac ventricles in response to increased wall tension [18,28], but it is also secreted by the brain, which is actually the organ where this substance was first identified [26]. Pro-BNP levels are increased in patients with acute stroke [32], but it is not well known if BNP is released by the ischemic brain or if this increase is due to previous of concurrent heart damage. Furthermore, the fact that BNP levels are higher in cardioembolic stroke independently of infarct volume, suggests that this increase may be related, at least in part, to a previous heart disease potentially associated with a cardiac source of embolism.

A recent study in patients with ischemic stroke found that increased pro-BNP levels are associated with electrocardiogram abnormalities suggestive of myocardial ischemia [12]. However, in our series we excluded patients with these abnormalities suggestive of myocardial ischemia in electrocardiogram. High levels of pro-BNP have also been associated with atrial abnormalities in patients with ischemic stroke, such as atrial dilatation, low flow velocity, spontaneous echocontrast or intraventricular thrombus, and atrial fibrillation [6]. Embolism of cardiac origin accounts for about $20 \%$ of ischemic strokes [22], and the majority of cardioembolic strokes are due to atrial fibrillation $[3,14$, 15,29]. Paroxysmal atrial fibrillation is an underdiagnosed arrithmia associated with an increased risk of ischemic stroke comparable to that of chronic atrial fibrillation $[4,15]$. Patients with paroxysmal atrial fibrillation show also higher levels of BNP [27], so these high levels in patients with stroke of undetermined origin might reflect the presence of paroxysmal atrial fibrillation or of any other atrial abnormality as potential cardioembolic source. 
Table 3

Basal characteristics of undetermined infarcts reclassified, univariated analysis

\begin{tabular}{|c|c|c|c|c|}
\hline & $\begin{array}{l}\text { Likely atherothrombotic } \\
n=22\end{array}$ & $\begin{array}{l}\text { Likely cardioembolic } \\
n=34\end{array}$ & $\begin{array}{c}\text { Undetermined } \\
n=26\end{array}$ & $\mathrm{p}$ \\
\hline Age, years & $60.0 \pm 14.3$ & $73.0 \pm 6.1$ & $71.0 \pm 9.5$ & $<0.0001$ \\
\hline Female, $\%$ & 27.3 & 47.1 & 30.8 & 0.245 \\
\hline History of hypertension, $\%$ & 27.3 & 64.7 & 53.8 & 0.022 \\
\hline History of diabetes, $\%$ & 18.2 & 17.6 & 15.4 & 0.961 \\
\hline Hypercholesterolemia, \% & 18.2 & 29.4 & 30.8 & 0.560 \\
\hline Smoker, $\%$ & 45.5 & 5.9 & 15.4 & 0.001 \\
\hline Alcohol consumption $>40 \mathrm{~g} /$ day, $\%$ & 27.3 & 0 & 0 & 0.001 \\
\hline Coronary heart disease, $\%$ & 0 & 0 & 7.7 & 0.110 \\
\hline Atrial fibrillation, $\%$ & 0 & 5.9 & 7.7 & 0.439 \\
\hline $\mathrm{SBP}, \mathrm{mm} \mathrm{Hg}$ & $146.2 \pm 28.1$ & $150.0 \pm 14.5$ & $158.4 \pm 12.9$ & 0.442 \\
\hline DBP, $\mathrm{mm} \mathrm{Hg}$ & $82.9 \pm 16.9$ & $79.8 \pm 13.3$ & $77.1 \pm 20.8$ & 0.177 \\
\hline Body temperature, ${ }^{\circ} \mathrm{C}$ & $36.4 \pm 0.3$ & $36.3 \pm 0.3$ & $36.1 \pm 0.2$ & 0.434 \\
\hline Glucose, $\mathrm{mg} / \mathrm{dL}$ & $122.9 \pm 32.1$ & $131.8 \pm 70.2$ & $141.3 \pm 24.1$ & 0.196 \\
\hline Fibrinogen, $\mathrm{mg} / \mathrm{dL}$ & $369.5 \pm 86.2$ & $371.5 \pm 114.5$ & $372.7 \pm 77.5$ & 0.995 \\
\hline Basal NIHSS & $8[5,10]$ & $14[9,16]$ & $12[5,19]$ & 0.004 \\
\hline DWI volume at admission, cc & $10.9[4.5,41.6]$ & $21.6[6.0,90.0]$ & $20.7[12.3,86.4]$ & 0.005 \\
\hline Carotid stenosis $>50 \%, \%$ & 18.2 & 0 & 0 & 0.003 \\
\hline Carotid stenosis $\leq 50 \%, \%$ & 81.8 & 0 & 0 & $<0.0001$ \\
\hline Intracranial stenosis, $\%$ & 18.2 & 11.8 & 0 & 0.093 \\
\hline Pro BNP, pg/mL & $45.2[15.1,159.2]$ & $1290.0[871.6,1692.2]$ & $183.9[121.8,570.7]$ & $<0.0001$ \\
\hline Pro-ANP, fmol/mL & $513.3[199.9,1064.4]$ & $2847.4[1271.5,6296.2]$ & $1299.3[848.3,4392.5]$ & $<0.0001$ \\
\hline $\mathrm{CK}-\mathrm{MB}, \mathrm{ng} / \mathrm{mL}$ & $2.5[1.9,4.3]$ & $3.2[2.3,4.9]$ & $3.6[2.1,4.9]$ & 0.564 \\
\hline Pro-BNP $\geqslant 360 \mathrm{pg} / \mathrm{mL}, \%$ & 0 & 94.1 & 30.8 & $<0.0001$ \\
\hline
\end{tabular}

SBP: systolic blood pressure, DBP: diastolic blood pressure, DWI: diffusion-weighted image, BNP: brain natriuretic peptide, ANP: atrial natriuretic peptide, CK-MB: creatin kinase-MB.

BNP manifest substancial biological variability [30] and multiple assays [2] exists for its determination. We have measured NT-proBNP by Elecsys 2010 (Roche), a method for measurement of pro-BNP clinically available, because several studies indicate that NT-proBNP testing has the same clinical utility as BNP [5,11,24]. Besides, NT-proBNP as measured by the Elecsys assay may be stored at $-20^{\circ} \mathrm{C}$ for at least four months without a relevant loss of the immunoreactive analyte [17]. This is an important point to reduce the interassay coefficient of variance. Another important point is that the biologic half-life for BNP is 20 versus 70 minutes for NT-proBNP and may be the result of differences in the clearance rate of these peptides from blood [31]. These details together with the fact that the lower value of biological variability was found for NT-proBNP support the validity of the results obtained in the study and suggested that the NTproBNP has more of an "averaging" effect, whereas BNP is more sensitive to acute changes in the disease processes

This study has a number of limitations. $\mathrm{CHADS}_{2}$ scale was designed to assess the embolic risk in patients with atrial fibrillation, but not in those with undetermined stroke. Few patients were undetermined by two causes, but in most of patients no atherothrombotic or embolic source was found. Only patients with undetermined stroke were subjected to $\mathrm{CHADS}_{2}$ and atherothrombotic risk scales, which could lead to bias, since these scales are not validated for the reclassification of undetermined stroke. The temporal profile of these molecular markers was not investigated in our study, and some studies found that pro-BNP levels decrease in the following days after stroke [32], so we cannot conclude if the increase of these molecular markers is the result of the ischemic lesion or reflects a previous cardiac damage. Other limitation is that we do not have information about previous treatment before admission that may affect pro-BNP levels.

In conclusion, we have found that serum levels of pro-BNP $>360 \mathrm{pg} / \mathrm{mL}$ within the first 24 hours from stroke onset may be useful to identify patients with an unknown cardiac source of cerebral embolism.

\section{Acknowledgements}

Partial results of this investigation were presented at the International Stroke Conference 2007 in San Francisco, USA (February 2007).

This project has been partially supported by grants from the Spanish Ministry of Health (Instituto de Salud Carlos III) RETICS-RD06/0026; the Consellería de Ed- 
ucación e Ordenación Universitaria of Xunta de Galicia (Axudas para a Consolidación e Estruturación de Unidades de Investigación Competitivas. Expediente: 80/2006); and Fundación de Investigación Médica Mútua Madrileña.

\section{References}

[1] H.P. Adams Jr., B.H. Bendixen, L.J. Kappelle et al., Classification of subtype of acute ischemic stroke. Definitions for use in a multicenter clinical trial, TOAST, Trial of Org 10172 in Acute Stroke Treatment, Stroke 24 (1993), 35-41.

[2] F.S. Apple, M. Panteghini, J. Ravkilde, J. Mair, A.H. Wu, J. Tate, F. Pagani, R.H. Christenson and A.S. Jaffe, Committee on Standardization of Markers of Cardiac Damage of the IFCC, Quality specifications for B-type natriuretic peptide assays, Clin Chem 51 (2005), 486-493.

[3] A. Arboix, L. García-Eroles, J.B. Massons, M. Oliveres, R. Pujades and C. Targa, Atrial fibrillation and stroke: clinical presentation of cardioembolic versus atherothrombotic infarction, Int J Cardiol 73 (2000), 33-42.

[4] C. Bell and M. Kapral, Use of ambulatory electrocardiography for the detection of paroxysmal atrial fibrillation in patients with stroke, Canadian Task Force on Preventive Health Care, Can J Neurol Sci 27 (2000), 25-31.

[5] M.R. Cowie and G.F. Mendez, BNP and congestive heart failure, Prog Cardiovasc Dis 44 (2002), 293-321.

[6] E. Di Angelantonio, S. De Castro, D. Toni, M.L. Sacchetti, F. Biraschi, M. Prencipe and M. Fiorelli, Determinants of plasma levels of brain natriuretic peptide after acute ischemic stroke or TIA, J Neurol Sci 260 (2007), 139-142.

[7] G. Fabinyi, D. Hunt and L. McKinley, Myocardial creatine kinase isoenzyme in serum after subarachnoid hemorrhage, $J$ Neurol Neurosurg Psychiatry 40 (1977), 818-820.

[8] B.F. Gage, A.D. Waterman, W. Shannon, M. Boechler, M.W. Rich and M.J. Radford, Validation of clinical classification schemes for predicting stroke: results from the National Registry of Atrial Fibrillation, JAMA 285 (2001), 2864-2870.

[9] G. Giannakoulas, A. Hatzitolios, H. Karvounis, G. Koliakos, A. Charitandi, T. Dimitroulas, C. Savopoulos, E. Tsirogianni and G. Louridas, N-terminal pro-brain natriuretic peptide levels are elevated in patients with acute ischemic stroke, Angiology 56 (2005), 723-730.

[10] L.E. Hackenberry, M.E. Miner, G.L. Rea, J. Woo and S.H. Graham, Biochemical evidence of myocardial injury after severe head trauma, Crit Care Med 10 (1982), 641-644.

[11] A. Hammerer-Lercher, E. Neubauer, S. Müller, O. Pachinger, B. Puschendorf and J. Mair, Head-to-head comparison of Nterminal pro-brain natriuretic peptide, brain natriuretic peptide and $\mathrm{N}$-terminal pro-atrial natriuretic peptide in diagnosing left ventricular dysfunction, Clin Chim Acta 310 (2001), 193-197.

[12] J.K. Jensen, L. Korsholm, P.F. Høilund-Carlsen, D. Atar, S.R. Kristensen and H. Mickley, The relation between electrocardiographic ST-T changes and NT-proBNP in patients with acute ischemic stroke, Scand Cardiovasc J 41 (2007), 294298.

[13] M. Kaste, H. Somer and A. Konttinen, Heart type creatine kinase isoenzyme (CK MB) in acute cerebral disorders, $\mathrm{Br}$ Heart J 40 (1978), 802-805.

[14] H.J. Lin, P.A. Wolf, E.J. Benjamín, A. Belanger and R.B. D'Agostino, Newly diagnosed atrial fibrillation and acute stroke, The Framingham Study, Stroke 26 (1995), 1527-1530.
[15] C. Marini, F. De Santis, S. Sacco, T. Russo, L. Olivieri, R. Totaro and A. Carolei, Contribution of atrial fibrillation to incidente and outcome of ischemic stroke: results from a population-based study, Stroke 36 (2005), 1115-1119.

[16] J. Montaner, M. Perea-Gainza, P. Delgado et al., Etiologic diagnosis of ischemic stroke subtypes with plasma biomarkers, Stroke 39 (2008), 2280-2287.

[17] T. Mueller, A. Gegenhuber, B. Dieplinger, W. Poelz and M. Haltmayer, Long-term stability of endogenous B-type natriuretic peptide (BNP) and amino terminal proBNP (NTproBNP) in frozen plasma samples, Clin Chem Lab Med $\mathbf{4 2}$ (2004), 942-944.

[18] M. Mukoyama, K. Nakao, K. Hosoda, S.I. Suga, Y. Saito, Y. Ogawa et al., Brain natriuretic peptide as novel cardiac hormona in humans: Evidence for an exquisite dual natriuretic peptide system, atrial peptide and brain natriuretic peptide, $J$ Clin Invest 87 (1991), 1402-1412.

[19] M. Naruse, Y. Takeyama, A. Tanabe et al., Atrial and brain natriuretic peptides in cardiovascular diseases, Hypertension 23 (1994), I231-I234.

[20] T. Naya, K. Yukiiri, N. Hosomi et al., Brain Natriuretic Peptide as a surrogate marker for cardioembolic stroke with paroxysmal atrial fibrillation, Cerebrovas Dis 26 (2008).

[21] J.W. Norris, V.C. Hachinski, M.G. Myers, J. Callow, T. Wong and R.W. Moore, Serum cardiac enzymes in stroke, Stroke $\mathbf{1 0}$ (1979), 548-553.

[22] S. Palacio and R.G. Hart, Neurologic manifestations of cardiogenic embolism, An update, Neurol Clin 20 (2002), 179-193.

[23] P.R. Puleo, P.A. Guadagno, R. Roberts, M.V. Scheel, A.J. Marian, D. Churchill and M.B. Perryman, Early diagnosis of acute myocardial infarction based on assay for subforms of creatine kinase-MB, Circulation 82 (1990), 759-764.

[24] A.M. Richards, M.G. Nicholls, T.G. Yandle, C. Frampton, E.A. Espiner, J.G. Turner, R.C. Buttimore, J.G. Lainchbury, J.M. Elliott, H. Ikram, I.G. Crozier and D.W. Smyth, Plasma $\mathrm{N}$-terminal pro-brain natriuretic peptide and adrenomedullin, New neurohormonal predictors of left ventricular function and prognosis after myocardial infarction, Circulation 97 (1998), 1921-1929.

[25] Y. Sato, H. Maruoka, Y. Honda, N. Hachiya and K. Oizumi, Plasma concentrations of atrial natriuretic peptide in cardioembolic stroke with atrial fibrillation, Kurume Med J 42 (1995), 71-77.

[26] T. Sudoh, K. Kangawa, N. Minamino and H. Matsuo, A new natriuretic peptide in porcine brain, Nature 332 (1988), 78-81.

[27] K. Tsuchida and K. Tanabe, Influence of paroxysmal atrial fibrillation attack on brain natriuretic peptide secretion, J Cardiol 44 (2004), 1-11.

[28] N. Valli, A. Gobinet and L. Bordenave, Review of 10 years of the clinical use of brain natriuretics peptide in cardiology, $J$ Lab Clin Med 134 (1999), 437-444.

[29] P.A. Wolf, R.D. Abbott and W.B. Kannel, Atrial fibrillation as an independent risk factor for stroke: the Framingham Study, Stroke 22 (1991), 983-988.

[30] A.H. Wu, A. Smith, S. Wieczorek, J.F. Mather, B. Duncan, C.M. White, C. McGill, D. Katten and G. Heller, Biological variation for N-Terminal Pro- and B-type natriuretic peptides and implications for therapeutic monitoring of patients with congestive heart failure, Am J Cardiol 92 (2003), 628-631.

[31] A.H. Wu, A. Smith, S. Wieczorek, J.F. Mather, B. Duncan, C.M. White, C. McGill, D. Katten and G. Heller, Biological variation for N-Terminal Pro- and B-type natriuretic peptides and implications for therapeutic monitoring of patients with congestive heart failure, Am J Cardiol 92 (2003), 628-631. 
[32] H.K. Yip, C.H. Sun, L.T. Chang, M.C. Chen and C.W. Liou, Time course and prognostic value of plasma levels of $\mathrm{N}$-terminal pro-Brain Natriuretic Peptide in patients after ischemic stroke, Circ J 70 (2006), 447-452.
[33] M. Yoshimura, H. Yasue, K. Okumura et al., Different secretion patterns of atrial natriuretic peptide and brain natriuretic peptide in patients with congestive heart failure, Circulation 87 (1993), 464-469. 


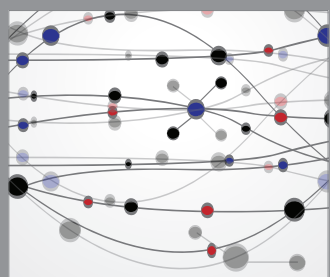

The Scientific World Journal
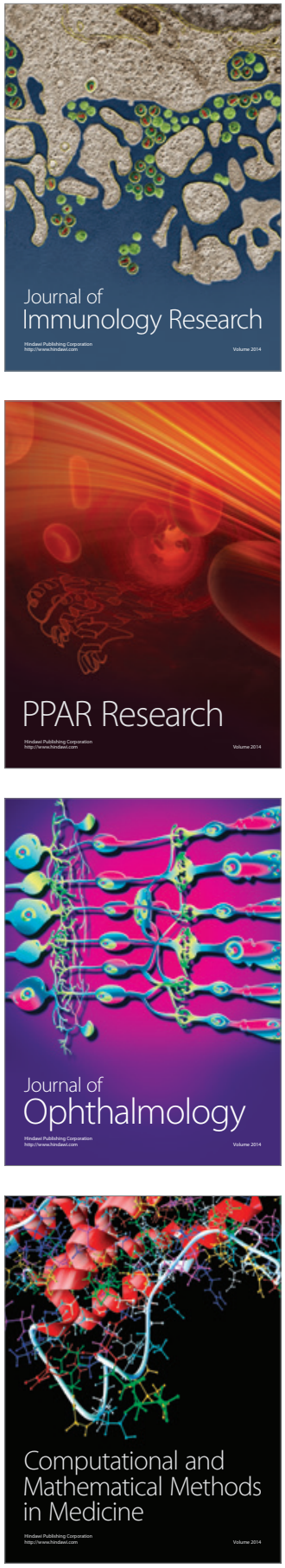

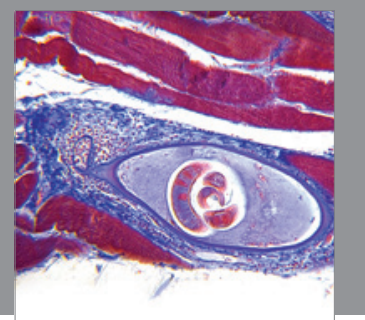

Gastroenterology

Research and Practice
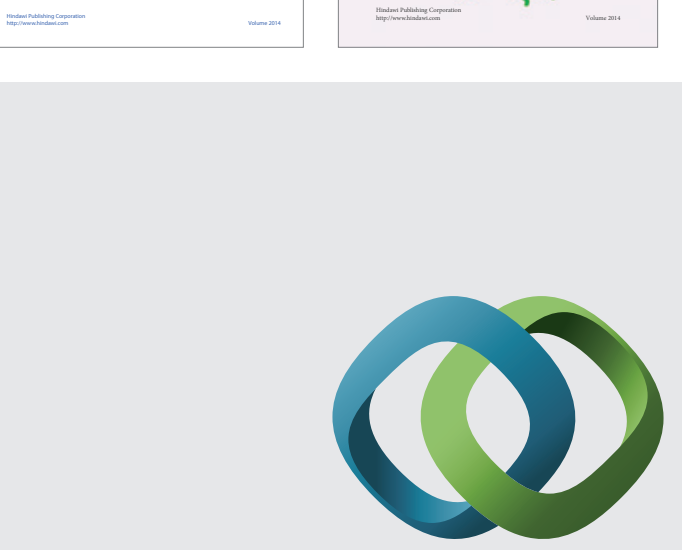

\section{Hindawi}

Submit your manuscripts at

http://www.hindawi.com
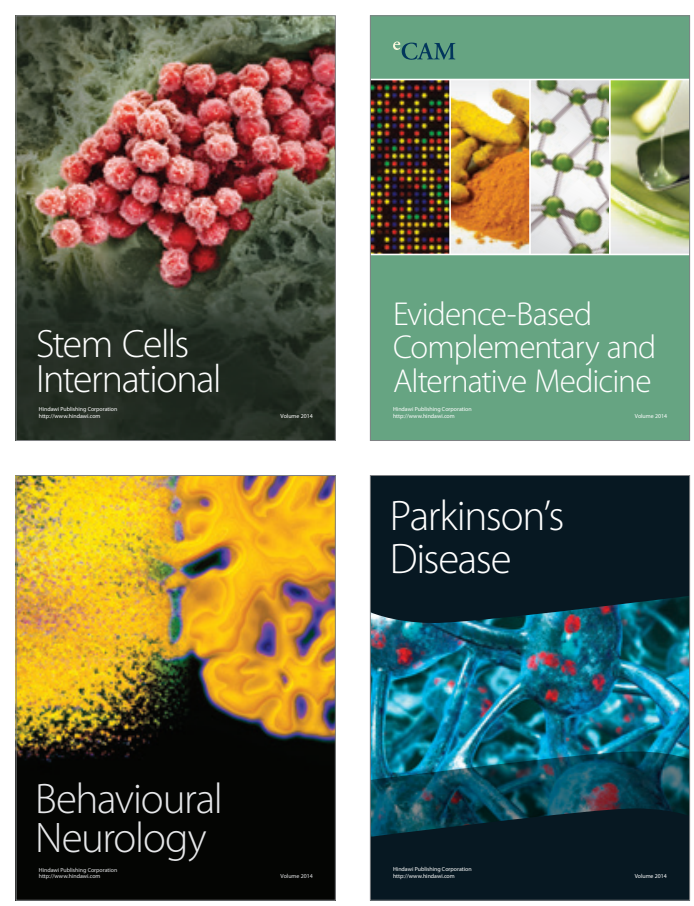

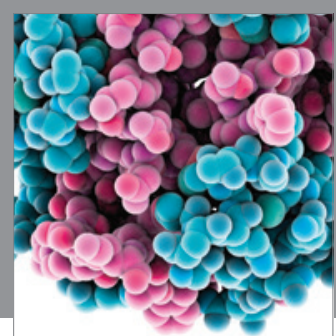

Journal of
Diabetes Research

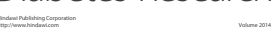

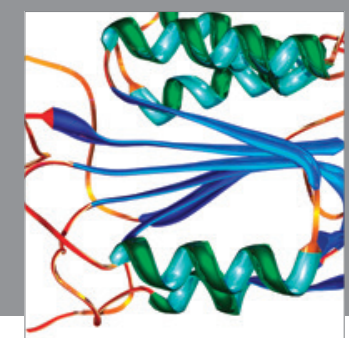

Disease Markers
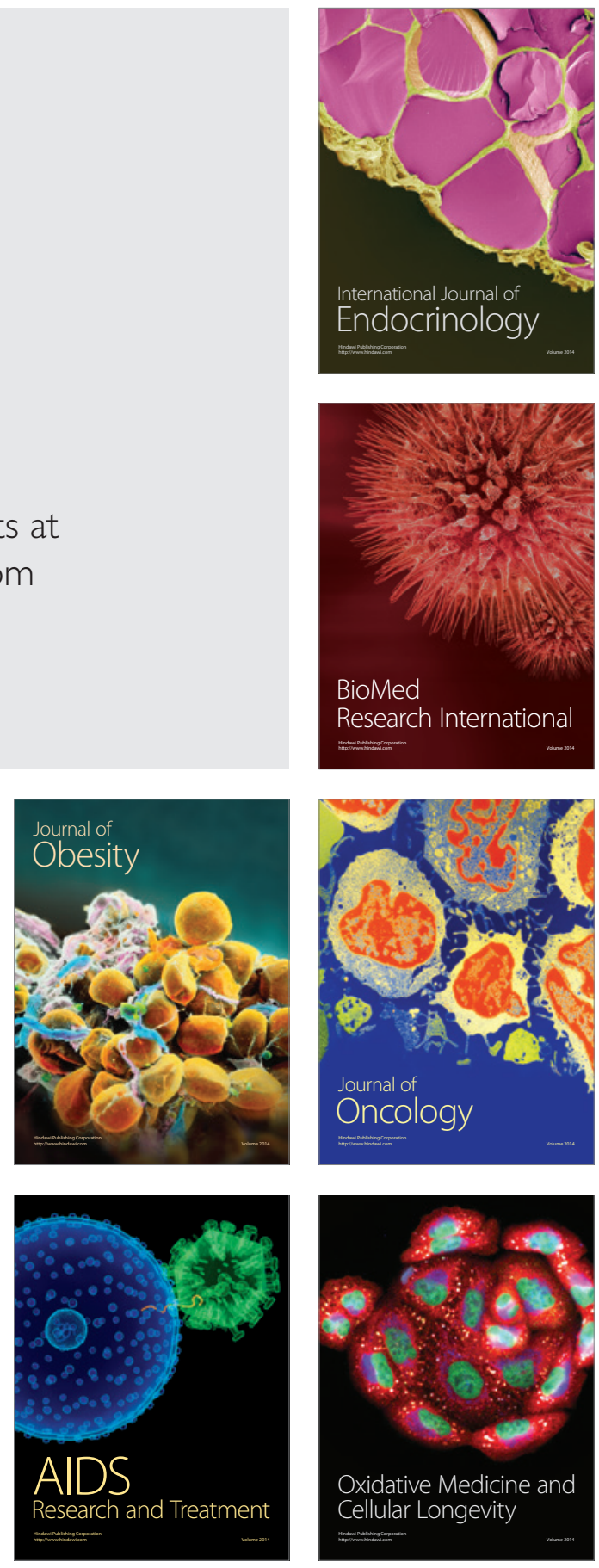